\title{
Ocean thermometer from the past
}

Noble gases dissolved in an ice core from Antarctica have revealed global mean ocean temperatures for 22,000-8,000 years ago with unprecedented accuracy, providing a crucial benchmark for refining climate models. SEE ARTICLE P.39

\section{RACHEL H. R. STANLEY}

$\mathrm{T}$ wenty thousand years ago, Earth was nearing the end of a glacial period. Gigantic ice sheets covered much of North America, Europe and Patagonia, air and water temperatures beyond the tropics were 4-23 degrees colder than today $^{1-3}$, depending on location, and atmospheric levels of carbon dioxide were approximately $35 \%$ lower $^{4}$. For reasons that are still unclear, the planet then transitioned to the warm, interglacial conditions that have lasted for about the past 11,000 years. On page 39 , Bereiter et al. ${ }^{5}$ report that noble gases trapped in an ice core from Antarctica provide a record of past mean ocean temperature during this transition, with unprecedented accuracy $\left( \pm 0.25^{\circ} \mathrm{C}\right)$ and high temporal resolution (250 years). This remarkable record will enable scientists to better formulate and update hypotheses on the transition between the last ice age and present-day warm conditions.

Much of the previously available information on ocean temperatures during the past thousands of years has come from records produced by organisms that lived in those times - for example, from differences in observed assemblages of the remains of marine biota ${ }^{6}$, from ratios of metal ions within preserved shells ${ }^{7}$, or from the arrangement of chemical bonds in lipid biomarkers called alkenones ${ }^{8}$, all of which have a known temperature dependency. The temperatures obtained from these records are valuable, but are subject to uncertainties due to the complex responses of the organisms to biological and environmental processes. As a result, these temperature proxies are typically accurate to approximately $1^{\circ} \mathrm{C}$. This is a problem, because the mean temperature change of the ocean is thought ${ }^{9}$ to have been only about $3^{\circ} \mathrm{C}$.

By contrast, Bereiter and colleagues used a technically challenging method ${ }^{10}$ in which noble gases trapped in an ice core (Fig. 1) act as

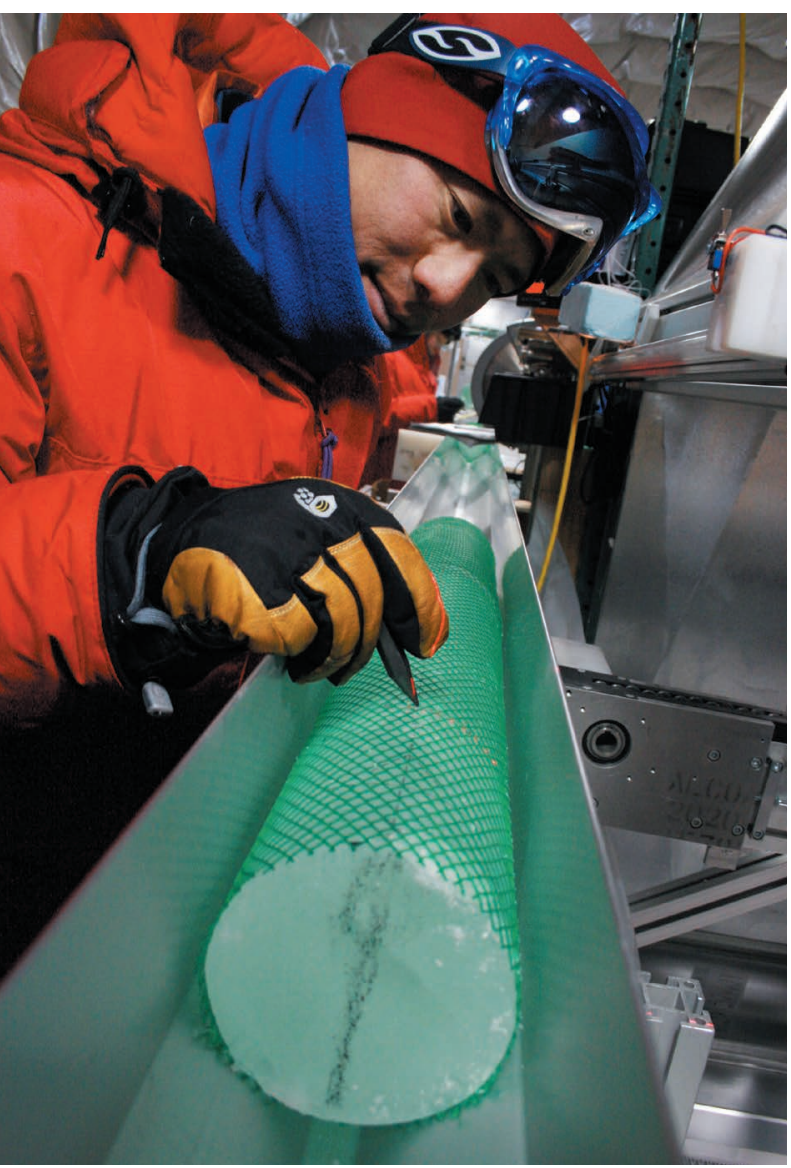

Figure 1 | Ice core from the West Antarctic Ice Sheet. Measurements of noble gases trapped in the ice core have been used to construct a ecord of global mean ocean temperatures $22,000-8,000$ years ago ${ }^{5}$.

in land ice thus provide a signal that can be used to deduce ocean temperature. Importantly, the laws that govern the physical processes underpinning this noble-gas proxy are more enduring than those that underpin the biological processes on which most other palaeotemperature proxies are based. Moreover, there is relatively little time delay between changes in ocean temperature and corresponding changes in the noble-gas signal, compared with many other proxies - the 'lag-time' of the noble-gas tracer is less than 100 years. Bereiter and colleagues' temperature record is therefore more accurate and has greater temporal resolution than other records.

The most valuable result of the authors' research is the temperature record itself, which scientists can use to test their climate models and hypotheses. For example, the record reveals that the temperature difference between the cold glacial period and the warm interglacial (up until the industrial period) was $2.57 \pm 0.24^{\circ} \mathrm{C}$, a number that models can now aim to replicate. Additionally, the high temporal resolution of the record means that model simulations can be checked at many time points during the transition, and can be used to explore interesting periods in the past in detail.

a proxy for temperature changes in the ocean. Noble gases are biologically and chemically inert, and therefore respond mainly to changes in physical conditions and processes, rather than in biological ones. In particular, the solubilities of noble gases - especially those of the heavier gases, such as krypton and xenon depend on temperature.

Gases are constantly exchanged between the ocean and atmosphere. As the ocean warms, krypton and xenon become less soluble in water, and so the ocean removes less of these gases from the atmosphere. The amount of krypton and xenon in the atmosphere therefore increases. The elemental and isotopic ratios of these elements in air bubbles trapped
The most surprising revelation from the temperature record is the extent of ocean warming during an event called the Younger Dryas, which occurred about 13,000-11,500 years ago. This event was an interruption in the overall warming trend, during which scientists think that temperatures dropped by a few degrees in the Northern Hemisphere ${ }^{11}$ but continued to increase, perhaps even at an accelerated rate, in the Southern Hemisphere ${ }^{12}$. Bereiter and colleagues report that the mean ocean temperature (which reflects the global ocean, but is weighted towards the Southern Hemisphere) increased substantially during the Younger Dryas, much more than had been estimated: the temperature increase was 
a whopping $1.6^{\circ} \mathrm{C}$ in only 700 years. This is about 1.7 times faster than the ocean is warming now because of global climate change. The reasons for this large warming should be investigated.

The authors also show that the ocean warmed faster than the atmosphere during the Younger Dryas, and then stopped warming before the atmosphere did. By contrast, there was a remarkable synchronicity between Antarctic air temperature, mean ocean temperature and atmospheric $\mathrm{CO}_{2}$ levels at all other times in the new record. Researchers must now find an explanation for the unusual asynchronicity during the Younger Dryas.

Bereiter and colleagues' work provides an unambiguous record of the average temperature of the entire ocean, from the surface to the greatest depths. However, it does not directly quantify surface temperatures - either global average surface temperature or sea surface temperatures, both of which are useful for understanding and quantifying glacial-interglacial temperature differences and processes. The authors do provide a rough estimate of average surface temperatures from their data, by using a cohort of models to estimate the ratio between sea surface temperature and mean ocean temperature. But this constrains surface temperatures only weakly, highlighting the need for more work in this area.

The authors present several fascinating hypotheses that stem from their data. For example, the observed synchronicity of mean ocean temperature with atmospheric $\mathrm{CO}_{2}$ levels and Antarctic air temperatures leads Bereiter et al. to conclude that the Southern Hemisphere drove Earth out of the glacial period. Furthermore, the large warming during the Younger Dryas suggests that changes in ocean dynamics beyond simple changes to the Atlantic Meridional Overturning Circulation (a climatically crucial component of ocean circulation) could be the cause of this climatic event. Climate modellers must now test these and other hypotheses by adding processes and feedbacks to their climate models, to see how the resulting ocean-temperature changes compare with those in the authors' noble-gasderived record. Much work will be needed to exploit the full potential of this beautiful record.

Rachel H. R. Stanley is in the Department of Chemistry, Wellesley College, Wellesley, Massachusetts 02481, USA.

e-mail:rachel.stanley@wellesley.edu

1. Dahl-Jensen, D. et al. Science 282, 268-271 (1998).

2. Cuffey, K. M. et al. Proc. Natl Acad. Sci. USA 113, 14249-14254 (2016)

3. Schneider von Deimling, T., Ganopolski, A., Held, H. \& Rahmstorf, S. Geophys. Res. Lett. 33, L14709 (2006).

4. Siegenthaler, U. et al. Science 310, 1313-1317 (2005).

5. Bereiter, B., Shackleton, S., Baggenstos, D., Kawamura, K. \& Severinghaus, J. Nature 553, 39-44 (2018).

6. CLIMAP Project Members. Science 191,
1131-1137 (1976).

7. Barker, S., Cacho, I., Benway, H. \& Tachikawa, K Quat. Sci. Rev. 24, 821-834 (2005).

8. Cacho, l. et al. Paleoceanography 16, 40-52 (2001)

9. Elderfield, H. et al. Science 337, 704-709 (2012)

10. Headly, M. A. \& Severinghaus, J. P. A. J. Geophys. Res. Atmos. 112, D19105 (2007)

11.Lea, D. W., Pak, D. K., Peterson, L. C. \& Hughen, K. A Science 301, 1361-1364 (2003).

12. Kaplan, M. R. et al. Nature 467, 194-197 (2010).

\section{CLINICAL NEUROSCIENCE}

\section{A bloody brake on myelin repair}

In multiple sclerosis, the blood-coagulation factor fibrinogen can enter the brain. It emerges that fibrinogen inhibits the maturation of cells called oligodendrocytes that repair nerve-fibre insulation and maintain neuronal communication.

\section{KLAUS-ARMIN NAVE \& HANNELORE EHRENREICH}

M ultiple sclerosis (MS) is a debilitating neurological disease in which the body's immune system destroys the myelin sheath that provides electrical insulation for nerve fibres. Myelin repair subsequently fails owing to a lack of new myelinproducing cells called oligodendrocytes, and this contributes to an irreversible loss of neuronal projections called axons. Why oligodendrocyte precursor cells (OPCs) located at sites of MS-related tissue damage fail to differentiate into oligodendrocytes has been poorly understood. Writing in Neuron, Petersen et al. ${ }^{1}$ report that a blood-coagulation factor called fibrinogen (which enters the brain when the blood-brain barrier is damaged in $\mathrm{MS}^{2}$ ) puts a brake on OPC differentiation. This insight offers hope for future treatment strategies.

Myelin is made by oligodendrocyte processes that spiral around axonal segments, and it forms a multilayered membrane sheath that speeds up electrical conduction. Oligodendrocyte processes also support axon metabolism. Myelin growth is a fast process in which oligodendrocyte mass multiplies in just a few days ${ }^{3}$. In mammals, myelination begins around birth and OPCs are maintained throughout life; myelination in the cortex of the adult brain is thought to contribute to learning and higher brain functions ${ }^{4}$. Orchestrating timely OPC generation, oligodendrocyte differentiation and energy-demanding myelin synthesis under changing metabolic conditions and in phases of physiological lowoxygen levels ${ }^{5}$ is a major challenge. Unsurprisingly, OPCs must integrate a plethora of external stimuli to determine when to differentiate.

Similarly, myelin repair following acute brain injury depends on optimal timing of OPC proliferation and differentiation. Unless cellular

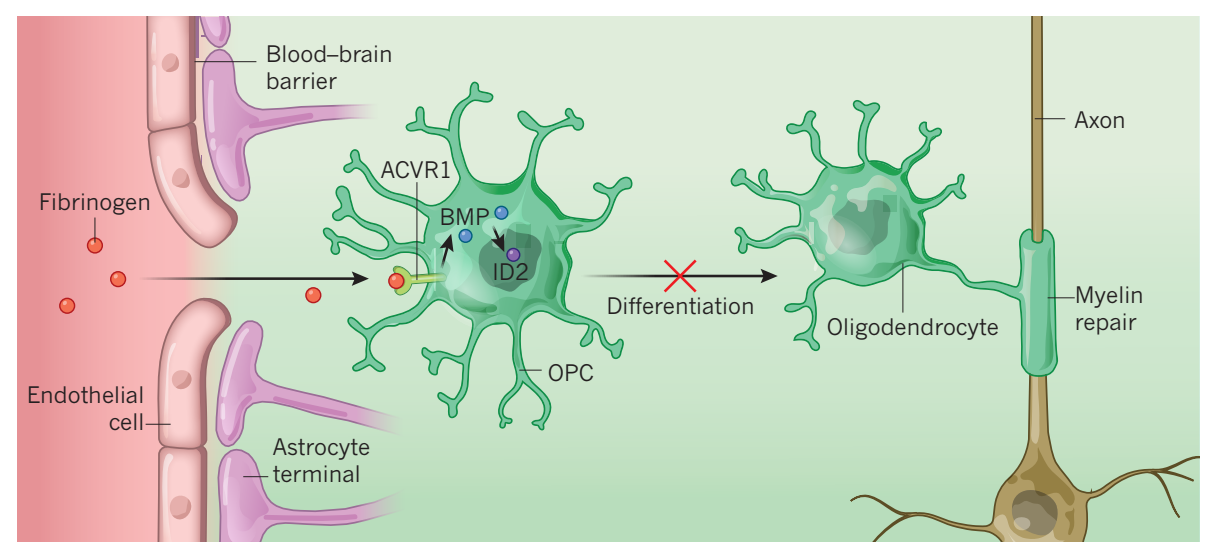

Figure 1 | A coagulation factor and multiple sclerosis (MS). In MS, neuronal projections called axons are stripped of their insulating myelin sheath. Subsequent myelin repair often fails, but the reason for this has been unclear. The blood-coagulation factor fibrinogen crosses the blood-brain barrier (composed of endothelial cells lined with the termini of cells called astrocytes) in MS, and Petersen $e t$ al. ${ }^{1}$ provide evidence that fibrinogen acts to inhibit myelin repair. They show that it binds to the receptor protein ACVR1 on the surface of oligodendrocyte precursor cells (OPCs), triggering an intracellular signalling cascade in which bone morphogenetic protein (BMP) activates the transcription factor ID2. BMP signalling prevents OPCs from differentiating into mature oligodendrocyte cells, which would produce myelin and so drive myelin repair. 